\title{
Whole-Day Gait Monitoring in Patients with Alzheimer's Disease: A Relationship between Attention and Gait Cycle
}

\author{
Maya Higuma $^{\mathrm{a}}$, Nobuo Sanjo ${ }^{\mathrm{a}, *}$, Hiroshi Mitoma ${ }^{\mathrm{b}}$, Mitsuru Yoneyama $^{\mathrm{c}}$ and Takanori Yokota ${ }^{\mathrm{a}}$ \\ ${ }^{\mathrm{a}}$ Department of Neurology and Neurological Science, Graduate School of Medical and Dental Sciences, \\ Tokyo Medical and Dental University, Tokyo, Japan \\ ${ }^{\mathrm{b}}$ Department of Medical Education, Tokyo Medical University, Tokyo, Japan \\ ${ }^{\mathrm{c}}$ MCHC R\&D Synergy Center, Inc., Kanagawa, Japan
}

Accepted 17 January 2017

\begin{abstract}
.
Background: Gait impairment in patients with Alzheimer's disease (AD) and its relationship with cognitive function has been described, but reports of gait analysis in $\mathrm{AD}$ in daily living are limited.

Objective: To investigate whether gait pattern of patients with $\mathrm{AD}$ in daily living is associated with cognitive function.

Methods: Gait was recorded in 24 patients with AD and 9 healthy controls (HC) for 24 hours by using a portable gait rhythmogram. Mean gait cycle and gait acceleration were compared between the AD and HC groups. For the AD group, these gait metrics were assessed for correlations with cognitive function, as determined by the Mini Mental State Examination and Wechsler Memory Scale-Revised (WMS-R).

Results: Although both gait parameters were not different between the patients with $\mathrm{AD}$ and $\mathrm{HC}$, gait cycle in patients with $\mathrm{AD}$ was positively correlated with attention/concentration scores on the WMS-R $(r=0.578)$, and not with memory function. Patients with AD with attention scores as high as $\mathrm{HC}$ displayed a longer gait cycle than both $\mathrm{HC}(p=0.048)$ and patients with $\mathrm{AD}$ with lower attention scores $(p=0.011)$. The patients with $\mathrm{AD}$ with lower attention scores showed a similar gait cycle with HC $(p=0.994)$.

Conclusion: Patients with AD with impaired attentional function walk with faster gait cycle comparable to $\mathrm{HC}$ in daily living walking, which was unexpected based on previous gait analysis in clinical settings. This result probably reflects diminished consciousness to either the environment or instability of gait in the patients with AD with impaired attention.
\end{abstract}

Keywords: Alzheimer's disease, attention, daily living, gait cycle, portable gait rhythmogram

\section{INTRODUCTION}

The major hallmark of Alzheimer's disease (AD) is progressive cognitive decline. Gait disturbance has been considered to occur only in advanced stages of the disease. However, quantitative gait analysis has shown that there are abnormalities in gait

\footnotetext{
*Correspondence to: Dr. Nobuo Sanjo, MD, PhD, Department of Neurology and Neurological Science, Graduate School of Medical and Dental Science, Tokyo Medical and Dental University, 1-5-45 Yushima, Bunkyo-ku, Tokyo, 113-8519 Japan. Tel.: +81 35803 5234; Fax: +81 35803 0169; E-mail: n-sanjo.nuro@ tmd.ac.jp.
}

performance in patients with $\mathrm{AD}$ even early in the disease course or the preclinical stage (mild cognitive impairment, MCI). Gait disturbances reported in early $\mathrm{AD}$ include slower gait with shorter stride length, lower cadence (longer stride time/gait cycle), and greater stride-to-stride variability [1-3]. These gait changes become apparent as cognitive decline progresses [3, 4]. Recently, gait analysis using a dual task paradigm, i.e., walking while simultaneously performing second task (cognitive task or motor task), has been widely used to evaluate cognitive involvement in gait control in patients with cognitive decline. It has been reported that dual task walking causes 
further decreases in gait velocity and stride length, and an increase in stride time and stride-to-stride variability when compared with usual walking in patients with AD [4-7]. Some previous reports have shown that dual-task related changes in those gait parameters are associated with global cognition, memory, and executive function [5, 7-10].

Although a dual task paradigm is used in gait performance tests to mimic walking in daily living, the subjects in the tests concentrate specifically on prescribed walking and cognitive tasks and environment is predictably arranged and their walking is supervised. Moreover, short-distance gait analyses might represent only limited aspects of gait in daily living, which demands a well-functioning ability to adapt walking behavior to much more complex environments. Therefore, long-time monitoring of gait can be useful to evaluate how people with dementia actually walk in their daily lives, and how cognitive function is associated with gait control under the circumstances of daily living.

The portable gait rhythmogram (PGR) is a recently developed acceleration sensor that detects tri-axial acceleration generated by movement of the trunk and limbs, and is capable of long-term monitoring of gait for up to 40 consecutive hours [11]. PGR quantifies gait acceleration (i.e. floor reaction force) and stride time of each stride from acceleration data. Gait assessments using the PGR have been reported in patients with Parkinson's disease (PD) [11-13] and in patients with cognitive decline [14]. Notably, consistency of mean gait cycle and mean gait acceleration obtained by the PGR over the course of 24 hours has been reported by a study that conducted 3 days of gait recording in a small number of patients with PD [13].

In this study, we measured gait cycle and gait acceleration using the PGR in 24 patients with AD and healthy controls, and also assessed their cognitive function. The purpose of this study was to investigate whether gait characteristics in daily living is different between patients with AD and healthy elderly, and whether gait parameters in patients with $\mathrm{AD}$ are also related to cognitive function in the daily living environment.

\section{MATERIALS AND METHODS}

\section{Subjects}

All patients with AD were recruited for this crosssectional study from the outpatient memory clinic of the Department of Neurology at Tokyo Medical and Dental University Hospital from July 2012 to February 2015. The diagnosis of probable AD was based on the National Institute of Neurologic and Communicative Disorders and the Alzheimer's Disease and Related Disorders Association (NINCDSADRDA) criteria. The inclusion criteria were as follows: (1) age $\geq 65$ years; (2) Mini Mental State Examination (MMSE) score $\geq 15$; (3) ability to walk without assistance or a walking aid in their daily living; (4) no other neurological diseases or orthopedic conditions affecting gait, major depression, or a concomitant cause of dementia. Twenty-four patients completed gait analysis and neuropsychological examination. Clinical diagnosis of AD has been not changed in any patients after mean follow up of $22.5 \pm 13.4$ months. Nine age-matched cognitive healthy subjects (healthy controls: $\mathrm{HC}$ ) were also recruited from the same outpatient clinic. The years of education and history of medication with drugs against dementia were recorded. History of falls during the past one year was also collected retrospectively from medical record.

The local ethical review board of Tokyo Medical and Dental University approved this study (approval number 982), and written informed consent was provided by all participants or their legal guardians.

\section{Gait analysis}

The PGR (Mimamori-gait system, LSI Medience Corporation, Tokyo, Japan) is a small device (size: $75 \times 50 \times 20 \mathrm{~mm}$, weight: $120 \mathrm{~g}$ ) that measures three-dimensional acceleration signals $\left(a_{x}, a_{y}, a_{z}\right)$ from voluntary movements of the trunk and limbs, and step-in and kick-off motion during gait [11]. The PGR was attached to the waist of the subjects, and subjects were instructed to wear this device all day (24 hours), except when changing clothes or taking a bath, and to perform their usual daily activities during gait recording.

The acceleration signals were recorded continuously at a sampling rate of $10 \mathrm{~ms}$. The data were automatically stored to a micro-SD card, and absolute values of the acceleration vectors $\left(a: a^{2}=a_{x}{ }^{2}+\right.$ $\mathrm{a}_{\mathrm{y}}{ }^{2}+\mathrm{az}_{\mathrm{z}}^{2}$ ) were calculated and graphically displayed on a personal computer $[11,15]$. "The amount of overall movements per 24 hour" was calculated as previously described [16]. Briefly, the accelerations induced by all truncal movement was averaged every $10 \mathrm{~min}$, and probability distribution of the acceleration was displayed graphically. "The amount of 
Table 1

Clinical features and cognitive characteristics of patients with $\mathrm{AD}$ and healthy controls $(\mathrm{HC})$

\begin{tabular}{lccc}
\hline Variable & $\mathrm{AD}(n=24)$ & $\mathrm{HC}(n=9)$ & $p$ value \\
\hline Age, years & $76.9 \pm 4.8$ & $74.6 \pm 4.4$ & 0.209 \\
Gender, male:female & $11: 13$ & $3: 6$ & 0.698 \\
Education, years $_{\text {Anti-dementia drug usage }}$ & $13.5 \pm 3.3$ & $14.2 \pm 2.1$ & 0.38 \\
History of fall (past 1 year) & $14 / 24(58.3 \%)$ & - & - \\
MMSE score & $5 / 17(29.4 \%)$ & $2 / 4(40 \%)$ & 1 \\
Raw index score of WMS-R & $22.5 \pm 3.0$ & $28.8 \pm 1.2$ & $<0.001$ \\
$\quad$ Verbal memory (0-97) & $21.5 \pm 11.3$ & $69.7 \pm 14.5$ & $<0.001$ \\
$\quad$ Visual memory (0-69) & $34.5 \pm 9.7$ & $58.7 \pm 2.8$ & $<0.001$ \\
$\quad$ Attention/concentration (0-88) & $53.3 \pm 7.4$ & $69.2 \pm 7.9$ & $<0.001$ \\
Delayed recall (0-106) & $14.2 \pm 14.8$ & $76.3 \pm 9.0$ & $<0.001$ \\
\hline
\end{tabular}

MMSE, Mini-Mental State Examination; WMS-R, Wechsler Memory Scale-Revised. The range of raw index scores is shown in parentheses. ${ }^{a}$ Acetylcholine esterase inhibitor or NMDA receptor antagonist (memantine).

overall movement" was defined by the mean value of this distribution, and have the dimension of $\mathrm{m} / \mathrm{s}^{2}[16]$. The acceleration vector generated by stepping was distinguished from those generated by other voluntary movements or artifacts based on mathematical methods of "template-matching (pattern matching)"; the exact analytical procedure has been described elsewhere $[13,15]$. In this study, the mean duration of the gait cycle (the time period from the contact of one foot with the ground to the next contact of the same foots) and the mean amplitude of gait acceleration, which is correlated with floor reaction force [11], throughout the 24 hours were used for analysis.

\section{Neuropsychological examination}

Cognitive function was assessed by the MMSE and Japanese version of the Wechsler Memory Scalerevised (WMS-R). The index scores (verbal memory, visual memory, attention and concentration, and delayed recall) were calculated from subtest scores of the WMS-R according to the manual [17]. Because the Japanese WMS-R has not been standardized for subjects over 75 years old, and $23(69.7 \%)$ of our subjects were over 75 years, we used the raw score of each index for analysis. Neuropsychological examinations were performed within 6 months of gait analysis.

\section{Statistics}

Results are reported as mean \pm SD. Because of the small number of the subjects, non-parametric statistics (Fisher's exact test, Mann-Whitney U-test) were used for comparisons between two groups, and Kruskal-Wallis ANOVA by ranks with Steel-Dwass post-hoc analysis was used for multiple comparisons between groups.
Because some of the cognitive parameters (MMSE, verbal memory, delayed recall) in the AD group did not follow a normal distribution, Spearman rank-order correlation was used to explore the relationship between gait parameters and each cognitive measure. Multivariate analysis adjusted for age or sex, history of falls and medication was performed when significant relationships between gait parameters and age (by Spearman correlation) or sex, history of falls, medication (by U-test) were observed. All statistical analyses were performed using EZR (Saitama Medical Center, Jichi Medical University), which is a graphical user interface for the R software environment (The R Foundation for Statistical Computing). A $p$-value of no more than 0.05 was considered statistically significant.

\section{RESULTS}

\section{Subjects}

There were no significant differences in age, sex, and education between the $\mathrm{AD}$ and $\mathrm{HC}$ groups (Table 1). All cognitive measures of MMSE and WMS-R were significantly lower in the patients with $\mathrm{AD}$. Ten of $24 \mathrm{AD}$ patients underwent gait and neuropsychological examinations before the prescription of anti-dementia medication. Information about history of falls was obtained from 17 patients with $\mathrm{AD}$, and $5 \mathrm{HC}$ subjects, and not different between two groups (Table 1).

\section{Gait parameters}

The mean gait cycle was $1.24 \pm 0.08 \mathrm{~s}$ in the $\mathrm{AD}$ group and $1.21 \pm 0.06 \mathrm{~s}$ in the $\mathrm{HC}$ group $(p=0.508)$. 


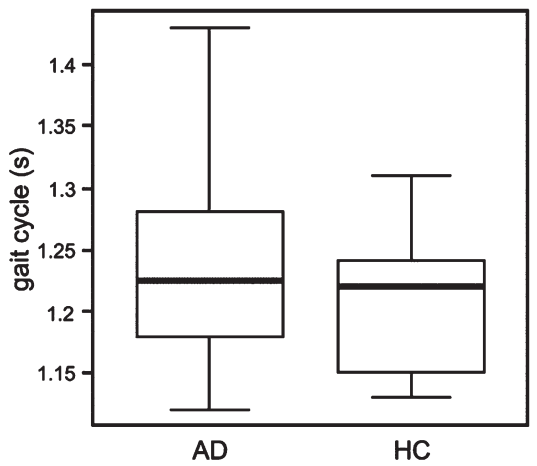

a

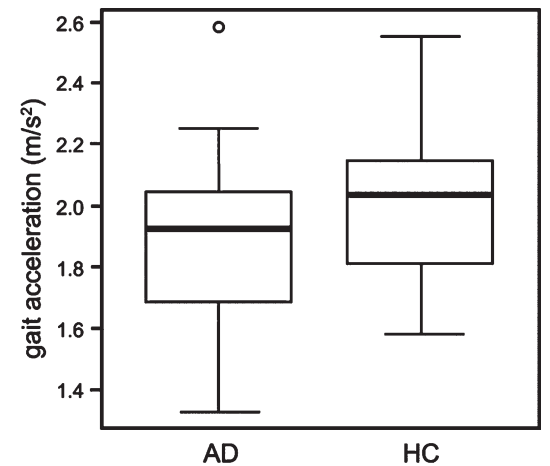

b

Fig. 1. Boxplots illustrating the comparison of gait cycle (a) and gait acceleration (b) among patients with Alzheimer disease (AD) and healthy controls (HC). The horizontal line inside the box indicates the median, and the length of the box is the interquartile range (IQR). The extremes of the whiskers contain the data within 1.5 IQR from the upper or lower quartile. The open circles indicate an outlier.

The mean gait acceleration was $1.90 \pm 0.27 \mathrm{~m} / \mathrm{s}^{2}$ in the AD group and $1.99 \pm 0.31 \mathrm{~m} / \mathrm{s}^{2}$ in the $\mathrm{HC}$ group $(p=0.417)$. Thus, these parameters were not significantly different between the two groups (Fig. 1). Amount of overall movement was $0.49 \pm 0.12 \mathrm{~m} / \mathrm{s}^{2}$ in the $\mathrm{AD}$ group and $0.50 \pm 0.12 \mathrm{~m} / \mathrm{s}^{2}$ in the $\mathrm{HC}$ group (Supplementary Figure 1a, $p=0.855$ ), indicating that the physical activity was not significantly different between two groups. In the AD group, gait acceleration was negatively correlated with age ( $r=-0.504, p=0.012$, Supplementary Figure 1b), but there was not an age-dependent difference in gait cycle $(r=-0.029, p=0.894)$. There was no obvious relation of sex, history of falls and anti-dementia medication with gait parameters (Supplementary Table 1).

\section{Relationship between gait parameters and cognitive function}

In the AD group, gait cycle was significantly correlated with the index score for attention/concentration $(r=0.578, p=0.033$, Fig. 2a filled circle), while gait acceleration showed a weak negative correlation with attention/concentration $(r=0.347, p=0.097)$. No obvious relationships between other cognitive measure and gait parameters were observed (Table 2). A multivariate analysis adjusted for age did not show any other significant relationship between gait acceleration and cognitive measures (data not shown).

Six patients had high attention/concentration scores corresponding to a healthy score, but this was different from other cognitive parameters. Therefore,
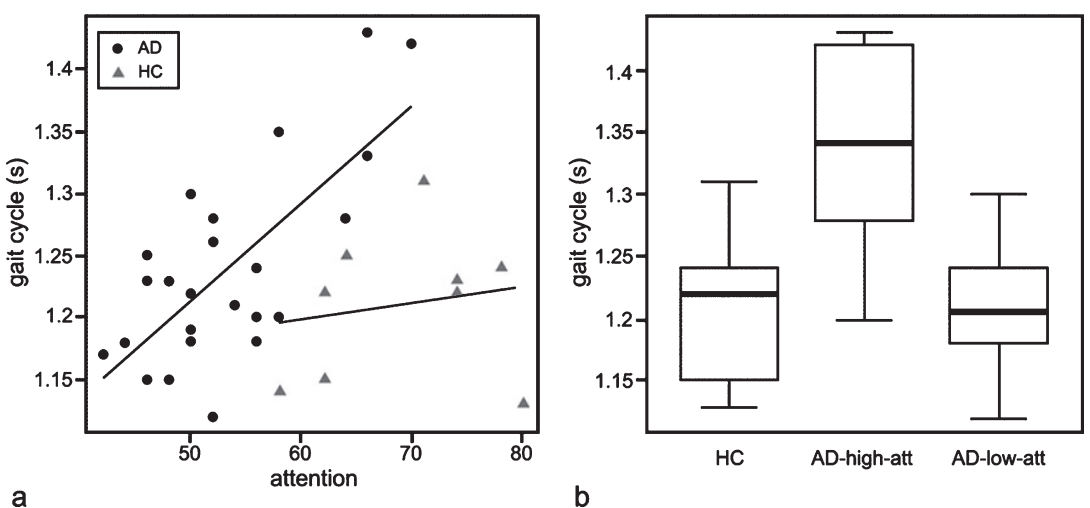

Fig. 2. (a) Scatter plots indicating the relationship between attention/concentration and gait cycle in patients with AD and healthy controls. Gait cycle and attention/concentration was moderately correlated in patients with AD, but not correlated in $\mathrm{HC}(p=0.101)$. The regression lines for $\mathrm{AD}$ (black line) and $\mathrm{HC}$ (gray line) are shown. (b) Boxplots showing a comparison of gait cycle between AD-high-att, AD-low-att, and HC. The parameters of these plots are the same as in Fig. 1. 
Table 2

Correlation between gait parameters and cognitive measures

\begin{tabular}{lrc}
\hline & \multicolumn{2}{c}{ Gait parameters } \\
\cline { 2 - 3 } & \multicolumn{1}{c}{ Gait cycle } & Gait acceleration \\
\hline MMSE & $-0.147(0.493)$ & $-0.289(0.171)$ \\
WMS-R & & \\
$\quad$ Visual memory & $-0.253(0.232)$ & $-0.040(0.852)$ \\
Visual memory & $-0.137(0.524)$ & $-0.058(0.789)$ \\
Attention/concentration & $0.578(0.003)$ & $-0.347(0.097)$ \\
Delayed recall & $-0.056(0.794)$ & $-0.042(0.844)$ \\
\hline
\end{tabular}

The Spearman correlation coefficients (r), with associated $p$-value in parentheses, are shown.

we divided the patients with $\mathrm{AD}$ into two groups (Table 3): an "AD-high-att" group in which attention/concentration scores were comparable with HC (i.e. within 1.5 SD of the mean HC score), and an "AD-low-att" group comprising patients with lower attention/concentration scores than HC. We compared gait cycle among these 3 groups using a Kruskal-Wallis ANOVA. The gait cycle in the ADhigh-att group was significantly longer than that in the AD-low-att $(p=0.011)$ and HC groups $(p=0.048$, Fig. 2b, Table 3), while the gait cycles in the AD-lowatt group and $\mathrm{HC}$ group were comparable.

\section{DISCUSSION}

In this study, we carried out long-term recordings of gait in patients with AD using PGR, and verified the possible relationship between gait and cognitive function. In spite of many previous reports that describe gait differences between patients with $\mathrm{AD}$ and healthy subjects, we did not find any difference in mean gait parameters between the two groups in this study. However, we found that gait cycle in patients with $\mathrm{AD}$ significantly correlated with attention/concentration function; the patients with attentional deficit walked with shorter gait cycle than the patients without discernible attentional deficit and the gait cycle of patients with attentional deficit was comparable to that of cognitively healthy subjects.

Involvement of attention/executive function in gait control has been reported in many correlation studies in patients with MCI and AD [5, 7-10], and in functional brain imaging studies related to gait performance $[18,19]$. Attentional dysfunction also correlates with increased risk of future falling in older adults [20]. Walking during a dual task paradigm test necessitates an ability to appropriately allocate attentional resources between walking and additional tasks. Therefore, subjects with lower attentional function exhibit deterioration of gait performance in dual tasking scenarios that generally manifests as slower gait speed, shorter stride length, longer stride time, and greater gait variability $[5,7,9,10]$. In a complicated external environment, these changes in gait pattern are observed even in healthy young people [21]. Therefore, it is natural to speculate that gait slowing in patients with lower attentional function would be more prominent than in subjects with normal attentional function in complex external environments encountered in daily living. However, the results of this study were in contrast to previous observations; indeed, we found that patients with $\mathrm{AD}$ with impaired attention walked at a faster pace in daily living than patients with preserved attention.

We suggest two possible explanations for this discrepancy from the viewpoint of attentional function. Firstly, in patients with significant attentional deficit, the manner in which limited attentional resources are allocated to gait, external environment, or other stimuli in testing situations might be much different than in daily living. In a testing environment, patients likely have high arousal and concentrate on prescribed tasks with supervision, while in daily living patients are more relaxed and not fully conscious

Table 3

Differences in gait cycle and attention score in patients with $\mathrm{AD}$

\begin{tabular}{|c|c|c|c|c|c|c|c|}
\hline \multirow[t]{2}{*}{ Variable } & \multirow{2}{*}{$\begin{array}{c}\text { AD-high-att } \\
(n=6)\end{array}$} & \multirow{2}{*}{$\begin{array}{c}\text { AD-low-att } \\
(n=18)\end{array}$} & \multirow{2}{*}{$\begin{array}{c}\mathrm{HC} \\
(n=9)\end{array}$} & \multicolumn{4}{|c|}{$p$-value ${ }^{\mathrm{a}}$} \\
\hline & & & & overall & $\begin{array}{l}\text { AD-high versus } \\
\text { AD-low }\end{array}$ & $\begin{array}{l}\text { AD-high } \\
\text { versus HC }\end{array}$ & $\begin{array}{l}\mathrm{AD} \text {-low } \\
\text { versus HC }\end{array}$ \\
\hline Age, years & $78.8 \pm 3.2$ & $76.2 \pm 5.1$ & $74.6 \pm 4.5$ & 0.177 & - & - & - \\
\hline Anti-dementia drug usage & $4 / 5(80 \%)$ & $9 / 18(50 \%)$ & - & - & $0.341^{b}$ & - & - \\
\hline MMSE & $23.2 \pm 23.2$ & $22.3 \pm 2.8$ & $28.8 \pm 1.2$ & $<0.001$ & 0.523 & $<0.001$ & $<0.001$ \\
\hline Attention/concentration & $63.7 \pm 4.8$ & $49.9 \pm 4.1$ & $69.2 \pm 7.9$ & $<0.001$ & $<0.001$ & 0.425 & $<0.001$ \\
\hline Gait cycle (s) & $1.34 \pm 0.09$ & $1.21 \pm 0.05$ & $1.2 \pm 0.06$ & 0.013 & 0.011 & 0.048 & 0.994 \\
\hline Gait acceleration $\left(\mathrm{m} / \mathrm{s}^{2}\right)$ & $1.79 \pm 0.16$ & $1.93 \pm 0.29$ & $1.99 \pm 0.31$ & 0.374 & - & - & - \\
\hline
\end{tabular}

${ }^{\mathrm{a}}$ Comparison among three groups were conducted by Kruskal-Wallis ANOVA by ranks with Steel-Dwass post-hoc analysis. ${ }^{\mathrm{b}}$ Comparison between two groups was conducted by Fisher's exact test. 
of the information or stimuli to which they should divide their attention. Subjects with relatively preserved attention are able to adopt their gait pattern according to the environment, even in complex contexts of daily living, just as they can in undertaking a dual-task gait test. Although we could not find any correlation between attention and gait cycle in our small number of HC subjects ( $r=0.101, p=0.795)$, a previous report on patients with non-demented Parkinson disease demonstrated that a relationship between attention executive function and gait pattern in daily living is compatible with clinical gait tests [22]. Conversely, the disproportionally shorter gait cycle observed in the patients with lower attention in the present study $(1.21 \pm 0.05 \mathrm{~s})$ was not much longer than that reported for single-task walking of $\mathrm{AD}$ patients in the literature [2, 6, 8, 23]. This may reflect gait not being significantly influenced by external environment in attentionally impaired people, or in other words, this may indicate carelessness towards the external environment.

Another, but more minor, possibility is the effect of so-called "cautious gait" [24]. Cautious gait is a form of higher-level gait disorder, and is characterized by a slowing of gait, shortened steps, mild widening of the base, and, according to recent quantitative gait analysis, longer gait cycle $[25,26]$. About $20 \%$ of the patients with $\mathrm{AD}$ and about $10 \%$ of healthy elderly individuals display this type of gait change [27]. Patients with $\mathrm{AD}$ often show increasing postural sway [28] and balance impairment [29]. Cautious gait is thought to be an appropriate adaptation of gait pattern to a consciously or subconsciously perceived instability or fear of falling [24, 25]. This adaptation of walking is considered to require various cortical functions including attention/executive function and integration of visual and sensory information $[19,30]$. It is possible that patients with $\mathrm{AD}$ with preserved attention can appropriately perceive their impaired balance, and adapt to their instability by taking cautious gait to avoid falling. On the contrary, patients with impaired attention may not be aware of instability in their gait, resulting in a faster gait cycle, which may even be as fast as healthy control individuals. A similar possibility was previously reported by van Iersel et al., who determined that the walking speed of elderly patients with dementia was disproportionally fast when adjusted for their overall degree of physical impairment. This phenomenon was attributed to frontal lobe disinhibition or lack of insight in demented subjects, and interpreted as an inappropriate adaptation to the environment [31].
Taken together, our study indicates that patients with AD with impaired attention tend to walk rather "carelessly", because of inappropriate allocation of limited (i.e. decreased) attentional resources to the external environment and/or poor self-awareness of their physical limitations or unsteadiness.

There may be various factors other than cognitive function that influence gait in daily living: physical condition, activity range, personal characteristics, supervision or guidance of activity by a caregiver, and so on. There was no significant difference in the total amount of movement between the two groups, although the specific activities that the subjects engaged in on the recording day were not assessed. How supervision and guidance of activity by others may have influenced gait pattern in our subjects is not clear; however, the impact of such biases on mean gait cycle throughout the day would not be expected to be very significant, as patients with severe dementia who always need help in daily living were not included in this study. Moreover, how individuals respond (e.g. change activity or gait) to intervention by others may to some extent depend on cognitive function.

Continuous gait monitoring in daily living in patients with $\mathrm{AD}$ has been performed in only few studies and seems to be difficult to interpret because gait disorder in $\mathrm{AD}$ may become apparent in only certain contexts of walking and other circumstances in daily living, which are complex and unpredictable. Nevertheless, we did observe a meaningful correlation between gait and cognitive function. To our best knowledge, this is the first study to show a relationship of a possibly inappropriately faster gait in patients with $\mathrm{AD}$ with impaired attention. This result may also bring new insights into fall risk in patients with AD. Previous studies showed that impairment in attention and executive function is related to fall risk in elderly people and patients with dementia $[18,20,32]$. The fall risk in subjects with impaired attention/executive function is generally discussed in relation to gait instability in dual-task paradigms [20, 33, 34]. We consider another possibility with respect to patients with $\mathrm{AD}$ : fall risk in those patients with attentional impairment may be attributable to their lack of ability to adapt their gait according to the environment and a resulting inappropriately faster gait in daily living. However, a prospective follow up study assessing linkage between falling risk, gait recording, and assessment of attentional function will be needed to verify this possibility. More generally, our study further demonstrates how long-term gait 
analysis is a useful tool for understanding real gait pattern in patients and how it is affected by different disease states.

In this study, we could not find differences in gait cycle between the AD group and the $\mathrm{HC}$ group. This mainly owes to the wide range of gait cycles in the $\mathrm{AD}$ group and relatively faster gait cycle in AD patients with declines in attentional capacity, as detailed above. Another factor is that the gait cycle of the HC group $(1.2 \pm 0.06 \mathrm{~s})$ was longer than that of healthy subjects in the clinical gait test (about 1.0 1.15 $\mathrm{s}$ in single or dual-task walking) $[2,6,8,23]$. This may also be explained by the effect of gait adaptation of $\mathrm{HC}$ subjects to various environments in daily living, but the change of gait cycle is smaller than in patients with $\mathrm{AD}$ with preserved attention, as indicated by clinical gait tests.

This study has some limitations. First, the number of subjects in our study was small especially for multiple subgroup analysis, thus necessitates caution.. Second, patients with AD in this study were limited to mild-to-moderate stages of disease, partly because only patients who could continuously wear the PGR for recording of gait for 24 hours and walk without any help or walking aid in daily living were selected. Another reason was that the patients with severe dementia were reported to show other types of gait impairment such as frontal gait [27]. Third, the cognitive batteries used in this study were mainly focused on memory function, and the indices for attention/concentration in the WMS-R reflect only a limited aspect of attention/executive function. Finally, we could not measure other gait parameters such as gait speed or stride length. Usually the gait cycle changes with gait speed and stride length in a regular pattern (i.e., as the gait become slower, the gait cycle become longer and stride length became shorter) $[35,36]$; however, the discrete effect of gait cycle and stride length on gait speed under different circumstances can vary. Thus, further evaluation is needed to verify the results of our study.

In conclusion, this study showed a correlation between attention and gait in daily living of patients with $\mathrm{AD}$; the correlation was opposite to the result of previous gait studies conducted under a conditioned environment. Gait slowing in daily living was observed in patients with preserved attention as expected. However, patients with impaired attention did not show significant gait slowing in daily living, implying that they walked with an inappropriately faster gait pattern in complex environments in daily living. Long-term gait analysis may give us further insight into understanding gait activity in actual daily living of patients with cognitive decline.

\section{ACKNOWLEDGMENTS}

We thank Yukiko Matsumoto and Yoshiko Miyama of our department for their help in conducting neuropsychiatric studies. We would like to thank Editage (http://www.editage.jp) for English language editing. There were no specific funding sources for this study.

\section{SUPPLEMENTARY MATERIAL}

The supplementary material is available in the electronic version of this article: http://dx.doi.org/ 10.3233/ADR-170001.

\section{REFERENCES}

[1] Allali G, Annweiler C, Blumen HM, Callisaya ML, De Cock AM, Kressig RW, Srikanth V, Steinmetz JP, Verghese J, Beauchet O (2016) Gait phenotype from mild cognitive impairment to moderate dementia: Results from the GOOD initiative. Eur J Neurol 23, 527-541.

[2] Nadkarni NK, Mawji E, Mcllroy WE, Black SE (2009) Spatial and temporal gait parameters in Alzheimer's disease and aging. Gait Posture 30, 452-454.

[3] Wittwer JE, Webster KE, Menz HB (2010) A longitudinal study of measures of walking in people with Alzheimer's Disease. Gait Posture 32, 113-117.

[4] Bridenbaugh S, Graham P, Wikson B, Ames D, Macaulay L, Martins R, Masters C, Maruff P, Rowe C, Szoeke C, AIBL, Ryan L, Allis K (2012) How does gait change as cognitive decline progresses in the elderly? Alzheimers Dement 8, 131-132.

[5] Sheridan PL, Solomont J, Kowall N, Hausdorff JM (2003) Influence of executive function on locomotor function: Divided attention increases gait variability in Alzheimer's disease. J Am Geriatr Soc 51, 1633-1637.

[6] Muir SW, Speechley M, Wells J, Borrie M, Gopaul K, Montero-Odasso M (2012) Gait assessment in mild cognitive impairment and Alzheimer's disease: The effect of dual-task challenges across the cognitive spectrum. Gait Posture 35, 96-100.

[7] Wittwer JE, Webster KE, Hill K (2014) The effects of a concurrent motor task on walking in Alzheimer's disease. Gait Posture 39, 291-296.

[8] Maquet D, Lekeu F, Warzee E, Gillain S, Wojtasik V, Salmon E, Petermans J, Croisier JL (2010) Gait analysis in elderly adult patients with mild cognitive impairment and patients with mild Alzheimer's disease: Simple versus dual task: A preliminary report. Clin Physiol Funct Imaging 30, 51-56.

[9] Doi T, Shimada H, Makizako H, Tsutsumimoto K, Uemura K, Anan Y, Suzuki T (2014) Cognitive function and gait speed under normal and dual-task walking among older adults with mild cognitive impairment. BMC Neurol 14, 67.

[10] Montero-Odasso M, Bergman H, Phillips NA, Wong CH, Sourial N, Chertkow H (2009) Dual-tasking and gait in people with mild cognitive impairment. The effect of working memory. BMC Geriatr 9, 41. 
[11] Mitoma H, Yoneyama M, Orimo S (2010) 24-hour recording of Parkinsonian gait using a Portable Gait Rhythmogram. Intern Med 49, 2401-2408.

[12] Terashi H, Utsumi H, Ishimura Y, Takazawa T, Okuma Y, Yoneyama M, Mitoma H (2012) Deficits in scaling of gait force and cycle in Parkinsonian gait identified by long-term monitoring of acceleration with the Portable Gait Rhythmogram. ISRN Neurol 2012, 306816.

[13] Terashi H, Utsumi H, Ishimura Y, Mitoma H (2013) Independent regulation of the cycle and acceleration in Parkinsonian gait analyzed by a long-term daily monitoring system. Eur Neurol 69, 134-141.

[14] Yoneyama M, Mitoma H, Higuma M, Sanjo N, Yokota T, Terashi H (2016) Ambulatory gait behavior in patients with dementia: A comparison with Parkinson's disease. IEEE Trans Neural Syst Rehabil Eng 24, 817-826.

[15] Yoneyama M, Kurihara Y, Watanabe K, Mitoma H (2014) Accelerometry-based gait analysis and its application to Parkinson's disease assessment. Part 1: Detection of stride event. IEEE Trans Neural Syst Rehabil Eng 22, 613-622.

[16] Utsumi H, Terashi H, Ishimura Y, Takazawa T, Hayashi A, Mochizuki H, Okuma Y, Orimo S, Takahashi K, Yoneyama M, Mitoma H (2012) Quantitative assessment of gait bradykinesia in Parkinson's disease using a portable gait rhythmogram. Acta Med Okayama 66, 31-40.

[17] Sugishita M (2001) Wechsler Memory Scale-Revised (Japanese), Nihon Bunka Kagakusya, Tokyo.

[18] Sheridan PL, Hausdorff JM (2007) The role of higher-level cognitive function in gait: Executive dysfunction contributes to fall risk in Alzheimer's disease. Dement Geriatr Cogn Disord 24, 125-137.

[19] Scherder E, Eggermont L, Swaab D, van Heuvelen M, Kamsma Y, de Greef M, van Wijck R, Mulder T (2007) Gait in ageing and associated dementias; its relationship with cognition. Neurosci Biobehav Rev 31, 485-497.

[20] Mirelman A, Herman T, Brozgol M, Dorfman M, Sprecher E, Schweiger A, Giladi N, Hausdorff JM (2012) Executive function and falls in older adults: New findings from a fiveyear prospective study link fall risk to cognition. PLoS One 7, e40297.

[21] Patterson MR, Whelan D, Reginatto B, Caprani N, Smeaton AF, Inomata A, Caulfield B (2014) Does external walking environment affect gait patterns? 36th Annu Int Conf IEEE Eng Med Biol Soc, pp. 2981-2984.

[22] Weiss A, Herman T, Giladi N, Hausdorff JM (2015) Association between community ambulation walking patterns and cognitive function in patients with Parkinson's disease: Further insights into motor-cognitive links. Parkinsons Dis 2015, 1-19.

[23] Allali G, Assal F, Kressig RW, Dubost V, Herrmann FR, Beauchet O (2008) Impact of impaired executive function on gait stability. Dement Geriatr Cogn Disord 26, 364-369.
[24] Nutt JG, Marsden CD, Thompson PD (1993) Human walking and higher-level gait disorders, particularly in the elderly. Neurology 43, 268-279.

[25] Herman T, Giladi N, Gurevich T, Hausdorff JM (2005) Gait instability and fractal dynamics of older adults with a cautious gait: Why do certain older adults walk fearfully? Gait Posture 21, 178-185.

[26] Kesler A, Leibovich G, Herman T, Gruendlinger L, Giladi N, Hausdorff JM (2005) Shedding light on walking in the dark: The effects of reduced lighting on the gait of older adults with a higher-level gait disorder and controls. J Neuroeng Rehabil 2, 27.

[27] O'Keeffe ST, Kazeen H, Philpott RM, Playfer JR, Gosney M, Lye M (1996) Gait Disturbance in Alzheimer's Disease: A clinical study. Age Aging 25, 313-316.

[28] Nakamura T, Meguro K, Yamazaki H, Okuzumi H, Tanaka A, Horikawa A, Yamaguchi K, Katsuyama N, Nakano M, Arai H, Sasaki H (1997) Postural and gait disturbance correlated with decreased frontal cerebral blood flow in Alzheimer disease. Alzheimer Dis Assoc Disord 11, 132139.

[29] Mazoteras Muñoz V, Abellan van Kan G, Cantet C, Cortes F, Ousset P-J, Rolland Y, Vellas B (2010) Gait and balance impairments in Alzheimer disease patients. Alzheimer Dis Assoc Disord 24, 79-84.

[30] Nutt JG (2013) Higher-level gait disorders: An open frontier. Mov Disord 28, 1560-1565.

[31] van Iersel MB, Verbeek ALM, Bloem BR, Munneke M, Esselink RAJ, Rikkert MGMO (2006) Frail elderly patients with dementia go too fast. J Neurol Neurosurg Psychiatry 77, 874-876.

[32] Herman T, Mirelman A, Giladi N, Schweiger A, Hausdorff JM (2010) Executive control deficits as a prodrome to falls in healthy older adults: A prospective study linking thinking, walking, and falling. J Gerontol Ser A Biol Sci Med Sci 65A, 1086-1092.

[33] Nakamura T, Meguro K, Sasaki H (1996) Relationship between falls and stride length variability in senile dementia of the Alzheimer type. Gerontology 42, 108-113.

[34] Hausdorff JM, Rios DA, Edelberg HK (2001) Gait variability and fall risk in community-living older adults: A 1-year prospective study. Arch Phys Med Rehabil 82, 1050-1056.

[35] Zijlstra A, de Bruin ED, Bruins N, Zijlstra W (2008) The step length-frequency relationship in physically active community-dwelling older women. Eur J Appl Physiol 104, 427-434.

[36] Merory JR, Wittwer JE, Rowe CC, Webster KE (2007) Quantitative gait analysis in patients with dementia with Lewy bodies and Alzheimer's disease. Gait Posture 26, 414-419. 